Research Article

\title{
The Structure of an Automatic Decision System for a Large Number of Independent Particle Detectors
}

\author{
Andreea Rodica Sterian \\ Academic Center of Optical Engineering and Photonics, Polytechnic University of Bucharest, \\ 313 Splaiul Independentei, 060042 Bucharest, Romania \\ Correspondence should be addressed to Andreea Rodica Sterian; andreea.sterian1@yahoo.com
}

Received 27 December 2012; Accepted 1 March 2013

Academic Editor: Bogdan Mitrica

Copyright (C) 2013 Andreea Rodica Sterian. This is an open access article distributed under the Creative Commons Attribution License, which permits unrestricted use, distribution, and reproduction in any medium, provided the original work is properly cited.

\begin{abstract}
The interest for large underground particle detector is increasing. Phenomena as proton decay and long base line neutrino oscillation are subject for many research projects over the world. Large detectors present also some problems regarding the large number of signals from independent photo multiplier tubes (PMTs). A realistic statistical model for numerical simulation of signal processing and sampling has been developed for the case of a large number of independent particle detectors (LNIPDs). Based on this analytical model of Poisson type, the structure of an automatic decision system based on the decision criterion of maximum a posteriori probability (MAP) or the maximum likelihood (ML) criterion is proposed. The purpose of the system is to analyze the exit from the measurement process and to decode the message transmitted, taking into account the presence of the noise which generates errors in the decoder. The system can be used later for detailed simulation of different types of huge underground particle detectors (like LAGUNA-LBNO experiment), where the large number of signals could become a real problem.
\end{abstract}

\section{Introduction}

We know that the abstract models are often used for the resolution of various problems. Starting from a detector response function that presents the relationship between the input factors and the output ones, we investigate a relaistic statistical model for numerical simulation of signal processing and sampling for the case of a large number of independent particle detectors (LNIPDs) and Poisson statistics of the detection process [1-6]. In the obtained equation, the statistical behaviour of the analyzed processes is essential. The basic criterion by which we can make a distinction between the different form of the detection process is the nature of the detection statistics which may be of Poisson type, Laguerre type, and so on. The nature of the detection statistics depends on the real situation by many factors as the number and size of the used detectors, the types of particles detected, the strength of the signal to be detected, and the extent by which the detected information needs to be processed after detection [7-14].
This paper proposes to use the mathematical model of Poisson detection based on the response function that correlates the random variables in a studied case of a large number of independent particle detectors (LNIPDs) providing a known type function of these complex processes. We define a response function and show that the measurement to detect $k$ particles in the interval $(t, t+T)$ is the type conditional Poisson. The further processing of the information obtained from the detection processes is based on decision criteria similar to the criteria from the information transmission theory. In this context, we propose a real-time method of automatic implementation of the decision-making process based on the information that results in the LNIPD [15-19].

In the paper, in order to process the data obtained from the study of the detection process in the LNIPD case, we proposed to use known decision criteria from the theory of information transmission. The method of automatic implementation of this decision process, which was described in this paper, based on the real-time information collected from LNIPD, is a major step forward in the implementation 
of the strategy proposed for the large underground particle detector implementation.

There are interconnections for which the optimal data analyses can be made automatically, in real time and in different conditions and situations. In the situations characterized by two random variables as in the case of LNIPD, the suitable statistical distribution is of the Poisson conditional type.

For an optimal and efficient settlement of the measurement equipments with minimum costs, automatic machines need to be built, which secure a real-time analysis and assure us of suitable correct decisions, being followed by adequate automatic data after detection processing [20-28].

\section{Detection Statistics}

Study of detection statistics represents an essential operation in the realization of an automatic decision system for large number of independent particle detectors (LNIPDs).

2.1. The Detector Response Function. Each electron makes a contribution to the output current which is converted into voltage by passing through the load resistance. Conversion process is a stochastic process in nature. From mathematical point of view, the output current is a superposition of the effects produced by each electron emitted. A single electron produces a current response function $h(t)$ :

$$
\int_{0}^{\infty} h(t) d t=e=1,6 \cdot 10^{-19} \mathrm{C} .
$$

A measurement done at the moment $t_{m}$ produces the response $h\left(t-t_{m}\right)$, so that in general we obtain the total response

$$
x(t)=\sum_{m=1}^{k(0, t)} h\left(t-t_{m}\right)
$$

where $k(0, t)$ represents the number of photoelectrons obtained in the interval $(0, t), t_{m}$ being the moment of measuring $m$. The process is called "counting." The response $x(t)$ depends on two random variables:

(i) random localizations of the measuring moments, $t_{m}$;

(ii) random values of the measured quantity, $k(0, t)$.

Because $t_{m}$ and $k(0, t)$ are random variables, $x(t)$ is a relatively complicated process to study because $x(t)$ does not explicitly contain the properties of the incident particles.

Therefore, we aim to find under what form the properties of the detected field are contained in the response signal and to highlight them.

2.2. Mathematical Model of the Detection Process. The model is represented by the probability that the detection process leads to $k$ photoelectrons in the interval $(t, t+T)$.

According to Fermi rule for the transition rate extended to that study, for a detector element $\Delta \vec{r}$, localized in point $\vec{r}$ on the radius of observer, we can see that the probability $P_{t}$ of an electron to be emitted by an element of area $\Delta \vec{r}$ located at the point $\vec{r}$ at the moment $t$ corresponds to the following transition rate $d P / d t$ :

$$
\frac{d P_{t}}{d t}=\alpha I(t, \vec{r}) \Delta \vec{r}
$$

where $I(t, \vec{r})$ is the intensity of the field at the detector situated in point $(\vec{r}, t)$ and $\alpha$ is a proportionality constant.

This problem consists of calculating the probability that all the LNIPD detectors emit $k$ electrons by their area $A$ in the interval $(t, t+T)$.

We must calculate composed probability in order to obtain $k$ electrons from all the LNIPD detectors.

It results $[8,22]$ that

$$
P_{k}(k)=\frac{\left(m_{v}\right)^{k}}{k !} \exp \left[-m_{v}\right] \quad k \geq 0,
$$

where, by definition,

$$
m_{v}=\alpha \int_{V} I(\vec{V}) d \vec{V}
$$

or

$$
m_{v}=\alpha \int_{A} \int_{t}^{t+T} I(\rho, \vec{r}) d \rho d \vec{r},
$$

$m_{v}$ being the probability level.

Therefore, (4) is also written under the following form [8]:

$$
\operatorname{Pos}\left(k, m_{v}\right)=\frac{\left(m_{v}\right)^{k}}{k !} \exp \left[-m_{v}\right],
$$

where $m_{v}$, the parameter of Poisson probability, is dimensionless.

According to (5), in the energetic space, $\alpha$ has the reversed size of energy.

The quantity $n(t, \vec{r})$ can be defined as "count intensity" by the relation

$$
n(t, \vec{r})=\alpha I(t, r),
$$

which represents a normalized intensity.

The integral of $n(t, \vec{r})$ gives directly the level of probability

$$
m_{v}=\int_{A} \int_{t}^{t+T} n(\rho, t) d \rho d \vec{r} .
$$

Alternately,

$$
n(t)=\int_{A} n(t, \vec{r}) d \vec{r}=\alpha \int_{A} I(t, \vec{r}) d \vec{r} .
$$

So,

$$
m_{v}=\int_{t}^{t+T} n(\rho) d \rho .
$$

It results that variable $k$ is a Poisson type.

The probability (7) is actually a conditional Poisson probability or double stochastic Poisson probability because it depends on $m_{v}$ which is a random variable (integral of 
a random variable). Therefore (in more precise case), $P_{k}(k)$ requires additional media over $m_{v}$ of $\operatorname{Pos}\left(k, m_{v}\right)$ as it can be considered a random variable with density $p_{m_{v}}(m)$ for $0 \leq$ $m \leq \infty$.

It results that

$$
\begin{aligned}
P_{k}(k) & =\int_{0}^{\infty} \operatorname{Pos}\left(k, m_{v}\right) p_{m_{v}}(m) d m \\
& =\int_{0}^{\infty} p_{m_{v}}(m)\left[\frac{m^{k}}{k !} e^{-m}\right] d m .
\end{aligned}
$$

\section{Processing of Information Obtained from LNIPD}

For the two types of emission, processes studied of Poisson type and Poisson conditioned type output level of the signal statistics are closely related as has been shown with intensity statistics.

Thus, formula (2) reveals proportionality of the photodetector output process with the emission of electrons.

Also, the formulas (5) and (8) show that for sufficiently large values of statistical intensity, the strength of the response of photodetector is determined basically by the incident field.

In order to process the data obtained from the LNIPD, the proposal of the paper is to use the known decision criteria from the theory of information. The method of automatic implementation of the decision process, which will be described later, based on the real-time information collected from the LNIPD, is a major step forward in the implementation of the automatic decision system in the case of a large number of independent particle detectors.

3.1. Characterization of Decision Criteria. The decisions are based on the detection statistics of photoelectrons, In the binary systems, we define the messages associated to binary symbols "one" or "zero" as bytes. Byte 1 is associated to the presence of the particle and byte 0 to the noise, in the absence of it.

\subsection{Formulation of Optimal Decision Criteria}

\subsubsection{Decision Criterion of Maximum A Posteriori Probability}

(MAP). According to this criterion,

(i) detectors measure the the incident field on the interval of a byte;

(ii) on the basis of detection statistics, we calculate the probability $P(i, \vec{k})$ of the particle incidence (byte $i$ ) when the vector $\vec{k}$ was detected;

(iii) it decides on the byte $i$ if:

$$
P(i, \vec{k})=\max P\left(\frac{j}{\vec{k}}\right)
$$

where $i, j=0,1$.
3.2.2. The Maximum Likelihood (ML) Criterion. According to this criterion,

(i) we define the plausibility function $\Lambda_{i}$ for byte $i$ by the relation

$$
\Lambda_{i}=P\left(\frac{\vec{k}}{i}\right)
$$

which means the probability of detecting vector $\vec{k}$ when particle $i$ is incident (byte $i$ ) on one photodetector;

(ii) we calculate the functions $\Lambda_{i}$;

(iii) we decide on byte $i$ if

$$
\Lambda_{i}=\max \left(\Lambda_{j}\right)
$$

In the considered case of binary decisions [8], it is expressed as equivalent as follows:

$$
\Lambda_{1} \geq \Lambda_{0}
$$

or

$$
\log \Lambda_{1} \geq \log \Lambda_{0}
$$

because the "log" function increases monotonically with its argument.

\section{Implementation of an Electronic Decoder for the Automatic Data Processing}

The role of MAP decoder (or ML) is to analyze the information resulted from LNIPD and to offer us correct data on the measuring process. The presence of different noise types determines errors in the decoder's decision.

4.1. Structure of Decoder in Case of Poisson Detection. We consider the distribution

$$
P\left(\frac{k_{j}}{i}\right)=\operatorname{Pos}\left(\frac{k_{j}}{\mu_{i j}+\mu_{b}}\right),
$$

where $\mu_{i j}$ is the "energy" number of the signal; $\mu_{b}$ is the average "energy" number of noise.

Equation (18) defines the probability that in the counting interval $j$ are measured $k_{j}$ when byte $i$ was realised.

From (18), $P\left(k_{j} / i\right)$ is explicitly written as follows:

$$
P(\vec{k}, i)=\prod_{j=1}^{2 B_{n} T} \frac{\left(\mu_{i j}+\mu_{b}\right)^{k_{j}}}{k_{j} !} \exp \left[-\left(K_{i}+K_{b}\right)\right]
$$

where $2 B_{n} T$ is the number of counting intervals in the time interval $[0, T], B_{n}$ is the signal bandwidth and $K_{i}, K_{b}$ are average numbers of signal and noise in the same interval $[0, T]$. 


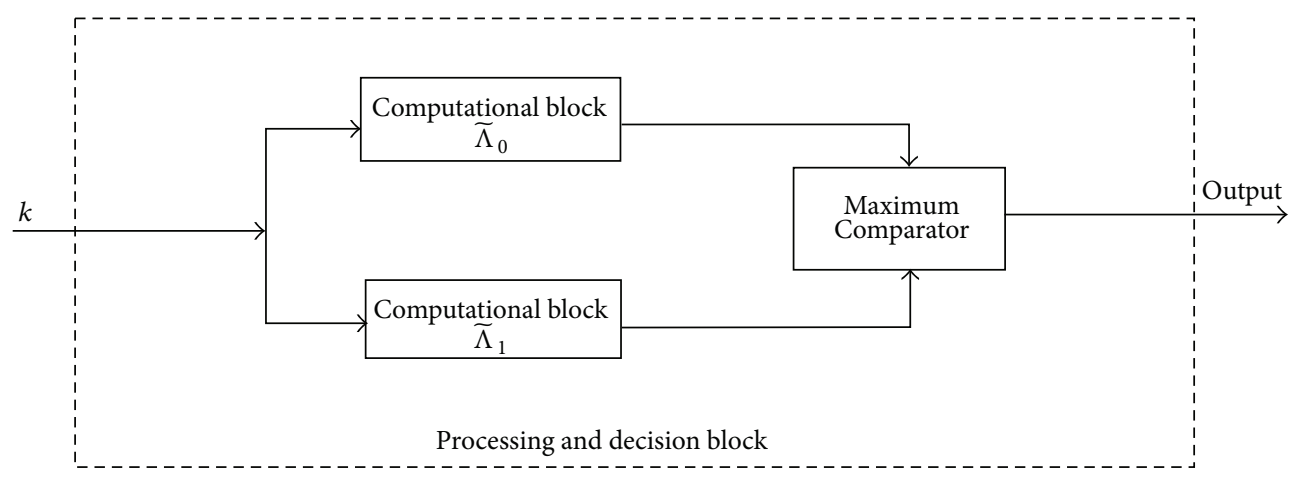

Figure 1: The block scheme of the processing and decision system.

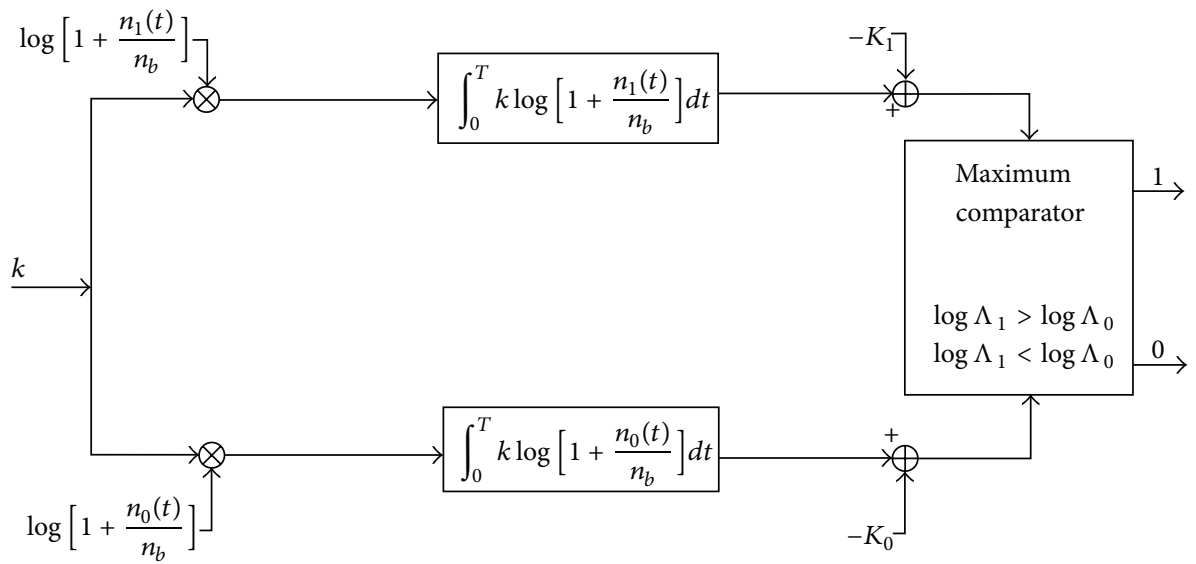

FIgURE 2: Integrated version of Poisson decoder.

9]

The expression of logarithmic plausibility function is [8,

$$
\begin{aligned}
\log \Lambda_{i}= & \sum_{j=1}^{2 B_{n} T} k_{j} \log \left(1+\frac{\mu_{i j}}{\mu_{b}}\right)-K_{i} \\
+ & {\left[\sum_{j=1}^{2 B_{n} T}\left(k_{j} \log \mu_{b}\right)-\log k_{j} !\right]-K_{b} . }
\end{aligned}
$$

The expression (20) can be simplified because the last bracket does not depend on $i$; so, it has the same value for both bytes $i=(0,1)$. Therefore, it can be neglected in the application of the decision test.

Consequently, we must evaluate only the quantity [5]

$$
\widehat{\Lambda}_{i}=\log \Lambda_{i} \cong \sum_{j=1}^{2 B_{n} T} k_{j} \log \left(1+\frac{\mu_{i j}}{\mu_{b}}\right)-K_{i}
$$

for $i=0,1$, and we compare the results.

The block scheme of the processing and decision system is presented in Figure 1.

The signals $K_{i}$ appear as a bias adjustment for the difference of energy in transmission of bytes.
4.2. Discrete Version of Poisson Decoder. In the structure, it has a pair of weighted summations circuits of detected signals according to expression (21), followed by a comparator of maximum.

Summations are discrete correlations of $\vec{k}$ to the corresponding logarithmic intensity vector. Because the maximal correlation function is the self-correlation, by the appropriate adjustment of polarizations $K_{1}$ and $K_{0}$, comparator of maximum determines correct decisions regarding the transmission of bytes " 1 " and " 0 ."

4.3. Integrated Version of the Poisson Decoder. The discrete version of the decoder is very appropriate for the operational analysis of this type of decoder. In the integrated version of decoder [8], summations are replaced with integration circuits (Figure 2).

\section{Conclusions}

The detector response function is used for obtaining the probability as that in the measuring process to determine $k$ photoelectrons in the interval $(t, t+T)$. This probability is of conditional Poisson type. For processing the data obtained from observing the detectors response, we have proposed the 
decision criteria to be used from the theory of information transmission. The method of automatic implementation of the decision process based on the data resulting in real time from LNIPD represents a very important step ahead in the implementation of the strategy proposed.

We have analysed the structure of an automatic decision system for the Poisson detection, based on the decision criteria (MAP or PM), known from information theory, in the discrete and integrated versions. For the modules of the block scheme, the electronic computation blocks were defined to be compatible with the blocks currently used in the data coding and decoding circuits; therefore, the practical implementation of such systems does not raise any problems. The methods of statistical physics we have discussed are inseparably part in the strategy for observations and measurements on the nuclear particles, as neutrinos. These observations provide us very important data about other low-mass particles which determine large-scale experiments in which these new types of particle detectors will be implemented. Concomitantly, we observe that a lot of work remains to be done to assure the accuracy of the experiments to be developed and built.

\section{References}

[1] C. Broggini, C. Giunti, and A. Studenikin, "Electromagnetic properties of neutrinos," Advances in High Energy Physics, vol. 2012, Article ID 459526, 47 pages, 2012.

[2] E. Ştefănescu, A. R. Sterian, and P. Sterian, "Study on the fermion systems coupled by electric dipol interaction with the free electromagnetic field," in Advanced Laser Technologies, Proceedings of SPIE, pp. 160-165, September 2004.

[3] D. A. Iordache, P. Sterian, F. Pop, and A. R. Sterian, "Complex computer simulations, numerical artifacts, and numerical phenomena," International Journal of Computers, Communications and Control, vol. 5, no. 5, pp. 744-754, 2010.

[4] A. G. Beda, V. B. Brudanin, V. G. Egorov et al., "The results of search for the neutrino magnetic moment in GEMMA experiment," Advances in High Energy Physics, vol. 2012, Article ID 350150, 12 pages, 2012.

[5] P. E. Sterian, Transmisia Optica a Informatiei, vol. 1-2, Technical Publishing House, Bucharest, Romania, 1981.

[6] L. N. Chang, Z. Lewis, D. Minic, and T. Takeuchi, "On the minimal length uncertainty relation and the foundations of string theory," Advances in High Energy Physics, vol. 2011, Article ID 493514, 30 pages, 2011.

[7] B. Mitrica, Studiul dependentei directionale a fluxului de miuoni si posibiltati de testare ale modelelor de interactie hadronica [Ph.D. thesis], University of Bucharest, Bucharest, Romania, 2010.

[8] R. M. Gagliardi and S. Karp, Optical Communications, WileyInterscience, New York, NY, USA, 1976.

[9] A. R. Sterian, "Coherent radiation generation and amplification in erbium doped systems," in Advances in Optical Amplifiers, P. Urquhart, Ed., InTech, Vienna, Austria, 2011.

[10] C. Cattani, "Fractional calculus and Shannon wavelet," Mathematical Problems in Engineering, vol. 2012, Article ID 502812, 26 pages, 2012.
[11] B. Mitrica, "Asymmetry of charge ratio for low energetic muons," AIP Conference Proceedings, vol. 972, no. 1, pp. 500504, 2008.

[12] M. Li, C. Cattani, and S.-Y. Chen, "Viewing sea level by a onedimensional random function with long memory," Mathematical Problems in Engineering, vol. 2011, Article ID 654284, 13 pages, 2011.

[13] C. Rosu, D. Manaila-Maximean, D. Donescu, S. Frunza, and A. R. Sterian, "Influence of polarizing electric fields on the electrical and optical properties of Polymerclay composite system," Modern Physics Letters B, vol. 24, no. 1, pp. 65-73, 2010.

[14] C. Cattani, S. Chen, and G. Aldashev, "Information and modeling in complexity," Mathematical Problems in Engineering, vol. 2012, Article ID 868413, 3 pages, 2012.

[15] H. Rebel, O. Sima, A. Haungs, C. Manailescu, B. Mitrica, and C. Morariu, "The muon charge ratio in cosmic ray air showers," Journal of Physics G-Nuclear and Particle Physics, vol. 35, no. 8, Article ID 085203, 2008.

[16] B. Mitrica, I. M. Brancus, H. Rebel et al., "Experimentally guided Monte Carlo calculations of the atmospheric muon and neutrino flux," Nuclear Physics B-Proceedings Supplements, vol. 151, no. 1, pp. 295-298, 2006.

[17] C. Cattani, "Harmonic wavelets towards the solution of nonlinear PDE," Computers and Mathematics with Applications, vol. 50, no. 8-9, pp. 1191-1210, 2005.

[18] P. E. Sterian, "Analytical model of dissipative coupling coefficients in laser structures," Proceedings of the Romanian Academy A, vol. 3, no. 3, pp. 123-126, 2002.

[19] C. Cattani and I. Bochicchio, "Wavelet analysis of bifurcation in a competition model," in Proceedings of the 7th International Conference on Computational Science (ICCS '07), vol. 4488 of Lecture Notes in Computer Science, pp. 990-996, 2007.

[20] A. Bercuci, B. Mitrica, M. Petcu et al., "Various types of measurements for atmospheric muons performed with the WILLI detector," Exotic Nuclei and Nuclear/Particle Astrophysics, 2006.

[21] P. E. Sterian, "Communications based on chaotic signals," Proceedings of the Romanian Academy A, vol. 3, no. 1-2, pp. 4548, 2002.

[22] O. Danila, P. E. Sterian, and A. R. Sterian, "Perspectives on entangled nuclear particle Pairs generation and manipulation in quantum communication and cryptography systems," Advances in High Energy Physics, vol. 2012, Article ID 801982, 10 pages, 2012.

[23] B. Sopori, T. Tan, and P. Rupnowski, "Photovoltaic materials and devices," International Journal of Photoenergy, vol. 2012, Article ID 673975, 1 pages, 2012.

[24] B. Mitrica et al., "Muon decay, a possibility for precise measurements of muon charge ratio in the low energy range $(<$ $1 \mathrm{GeV} / \mathrm{C})$," in Proceedings of the International Workshop on New Applications of Nuclear Fission, pp. 190-192, Bucharest, Romania, September 2003.

[25] C. Morariu, C. Manailescu, H. Rebel, O. Sima, A. Haungs, and B. Mitrica, "The muon charge ratio in cosmic ray air showers," Nuclear Physics B-Proceedings Supplements, vol. 196, no. C, pp. 414-417, 2009.

[26] B. Mitrica, "20 years of cosmic muons research performed in IFIN -HH," AIP Conference Proceedings, vol. 1498, pp. 291-303, 2012.

[27] B. Mitrica, M. Petcu, A. Saftoiu et al., "Investigation of cosmic ray muons with the WILLI detector compared with the predictions of theoretical models and with semi-analytical formulae," 
Nuclear Physics B-Proceedings Supplements, vol. 196, no. C, pp. 462-465, 2009.

[28] I. Lazanu et al., "About the possibility to measure some standard model parameters and search for new physics with low energy neutrinos," Romanian Reports in Physics, vol. 64, no. 1, pp. 2432, 2012. 

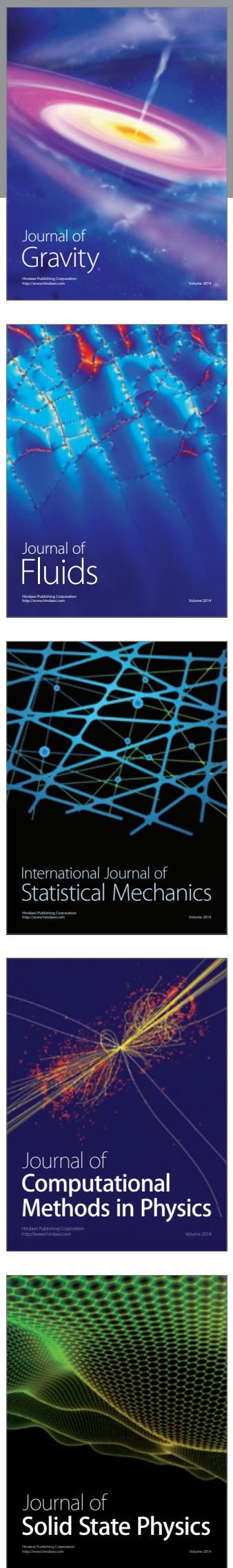

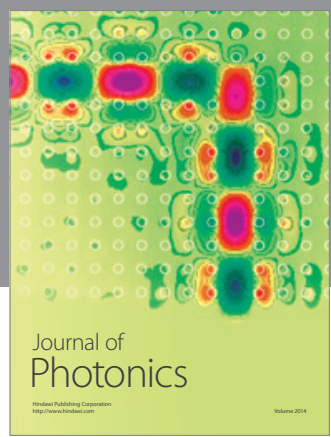

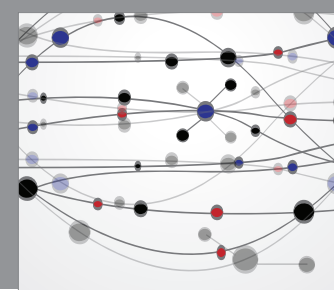

The Scientific World Journal

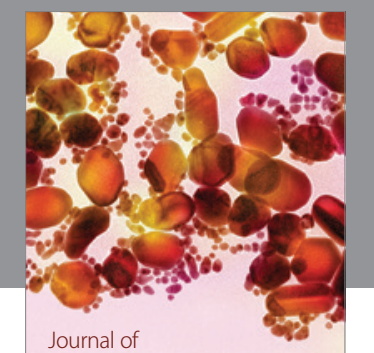

Soft Matter
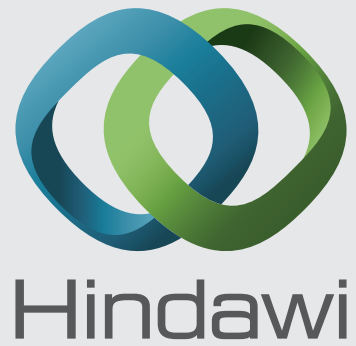

Submit your manuscripts at

http://www.hindawi.com
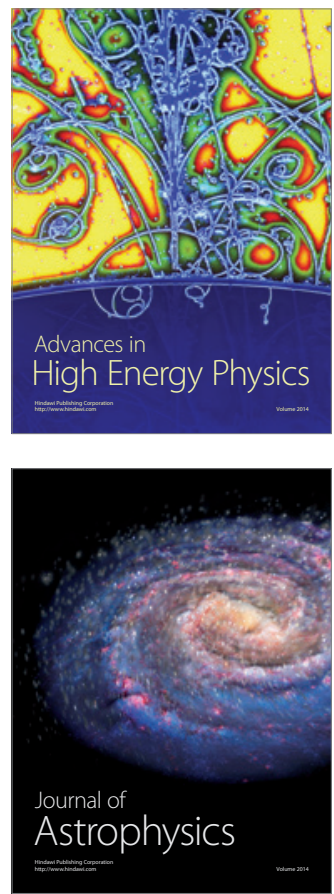
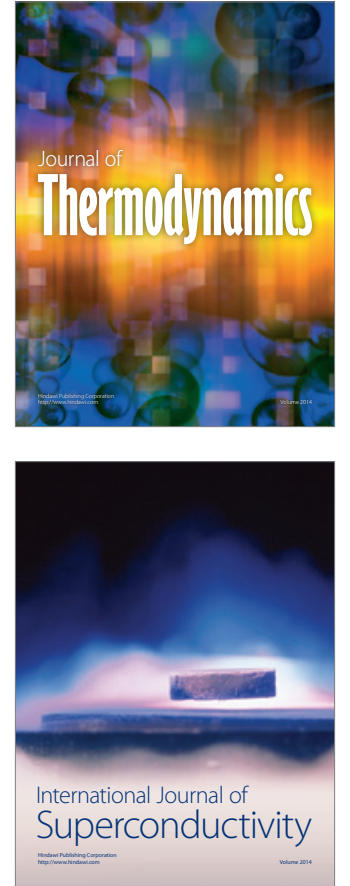
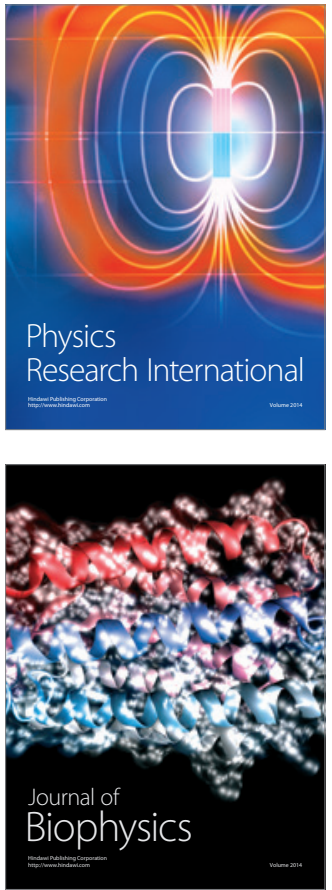
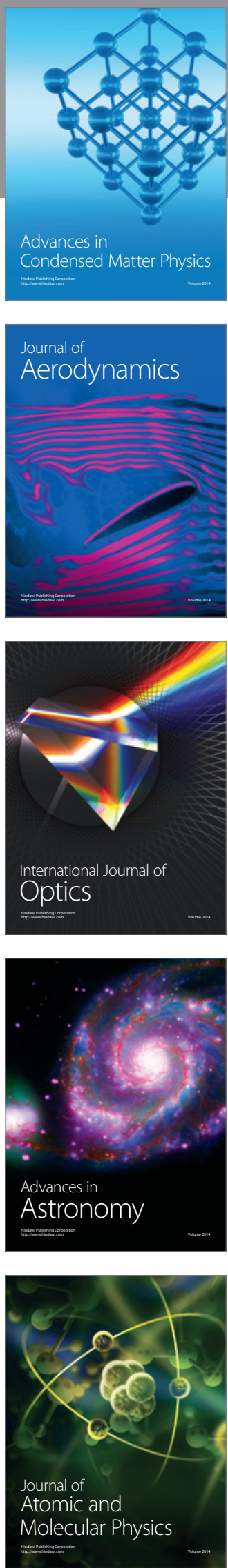